\title{
Knowledge and utilization of preventive measures in the control of neonatal malaria in south-eastern Nigeria
}

UCHECHUKWU M. CHUKWUOCHA*, GEOFFREY C. NWAKWUO and LAWRENCE O. ALINNOR

Department of Public Health Technology, School of Health Technology, Federal University of Technology, P.M.B 1526, Owerri, Imo State, Nigeria

\begin{abstract}
Background: The burden of neonatal malaria remains a major public health problem in Nigeria receiving less attention. Knowledge and awareness of preventive measures of neonatal malaria is still very low. This study aimed at assessing the Knowledge and utilization of preventive measures in the control of neonatal malaria in South-eastern Nigeria.

Methods: Structured pretested questionnaires were used to collect data from 230 consenting pregnant women attending antenatal clinics in the Federal Medical Centre, Owerri, Nigeria. These women were followed up until delivery and blood samples were taken from their new born for malaria parasite tests.

Results: There was high level of awareness on the use of malaria preventive measures by pregnant women in the control of neonatal malaria. A significant relationship between the use of malaria preventive measures and neonatal malaria was also shown. Over three-quarters (79.7\%) of women had knowledge of malaria preventive measures during pregnancy. Use of anti-malarial prophylaxis (91.8\%) followed by the use of insecticide treated nets (ITNs) (49.5\%) were practiced. ITNs were less (19.6\%) used during pregnancy. Most of them (57.8\%) received single dose of intermittent preventive treatment (IPT) using sulphadoxinepyrimethamine during pregnancy, while $37 \%$ received two doses of IPT.

Conclusion: More effort need to be made and support provided to women during pregnancy to encourage usage of preventive measures. There is also need to develop a health promotion package to eliminate factors discouraging the use of specific preventive measures like IPTp and ITNs.
\end{abstract}

Keywords: neonatal malaria, preventive measures, utilisation, women, knowledge, Nigeria

\section{Introduction}

The burden of Plasmodium falciparum malaria in children aged under five years has been well documented (WHO, 2015), however information on the burden of malaria in early infancy especially the neonatal period is scarce, inconclusive and contradictory, with a wide range of results reported (Runsewe et al., 2006; Ekannem et al., 2008; Larru et al., 2009; D'Alessandro et al., 2012). Febrile illnesses in neonates are most times attributed to septicaemia (Lopez et al., 1997; Kevalas, 2005; Pusic, 2007) and therefore the possible involvement of malaria at that point is not adequately considered. This is because neonatal malaria is thought to be rare due to the protective effect of maternal immunity after birth. However, reports have indicated that this protection may not be complete and that malaria can actually have transferred to the new born eventually leading to severe disease (Ekannem et al., 2008; D'Alessandro et al., 2012).

Despite the documented effects of malaria on the foetus and newborn, the specific role of preventive measures in the burden of neonatal malaria is not given adequate attention in Nigeria and other endemic areas. In Nigeria, data on neonatal malaria is lacking. However, the World Health Organization (WHO) has developed guidelines for the control of malaria during pregnancy in Sub-Saharan Africa (Mendenez et al., 2007). These consist of prompt and effective case management of malaria illness, combined with prevention of infection and/or disease by insecticide-treated nets (ITNs) and intermittent preventive treatment in pregnancy (IPTp) (WHO, 2004; Nosten et al., 2007).

The benefits of malaria preventive measures to both mother and the newborn in SubSaharan Africa has been reported (Gamble et al., 2006). Use of ITNs and prompt and effective

${ }^{*}$ Correspondence Email: uchukwuocha@gmail.com 
case management of malaria have been recommended by WHO as a three pronged approach to the prevention and control of malaria during pregnancy in areas of stable transmission (WHO, 2004). Utilization of preventive measures by pregnant women have the added benefit that they continue to protect the newborn baby during early infancy (Steketee et al.,2008). In addition to ITN at household level, other protective measures include protective clothing, insect repellents and spraying of insecticides (Yusuf et al., 2008).

However, despite all these efforts, it seems that mothers especially those in the rural areas are not abreast with the need and benefits of taking preventive measures during and after pregnancy against neonatal malaria. It is very important to note that the level of knowledge and awareness in this respect might still be very low. Thereby leading to very low compliance and utilization. Other factors which are social and economical in nature may also play important roles. This study was therefore aimed at assessing the knowledge and utilization of preventive measures in the control of neonatal malaria in South-eastern Nigeria.

\section{Materials Methods}

\section{Study setting and sampling of study subjects}

The study was conducted at Federal Medical Centre, Owerri, Nigeria which is a major health care facility serving as a referral centre while at the same time undertaking primary health care functions. Owerri is located in the tropical rain forest with climatic and environmental conditions that support malaria endemicity. This study employed a descriptive cross-sectional study design. A systematic random sampling technique was used to select a total of 230 pregnant mothers who presented at the obstetric unit of the health facility. This selection was done by simple balloting over a period of six Months among about 604 pregnant women that presented at the facility during the period.

\section{Data collection}

These women were followed up till delivery and till when blood samples could be collected from their new-borns for malaria parasite tests. Structured pretested questionnaires were administered to the study subjects to collect data on their knowledge and utilization of preventive measures in the control of neonatal malaria as well as their socio-clinical data. Blood samples were collected from the neonates by heel prick, thick films were made followed by Giemsa staining and microscopic observation to confirm the presence of malaria parasites.

\section{Data analysis}

Data generated were sorted out, coded and imputed into IBM SPSS version 20. Frequency tables and graphs were generated for relevant variables. Quantitative variables were summarized with proportions and chi-square test was used to determine associations between qualitative variables.

\section{Ethical consideration}

The study was approved by the Ethical Committee of the Federal Medical Centre Owerri, Nigeria. Informed oral consent was sought and obtained from the study subjects after a full explanation of the study was made them.

Results

The mean age + standard deviation the women was $29.1 \pm 5.4$ years, with majority of them (83.1\%) aged between 20-35 years. Three quarter of them had tertiary education and all the women were Christians. Ninety-two (40.3\%) were multigravidae, while 84 (36.4\%) of them were primigravidae. Over three quarters of the women $(79.7 \%)$ had heard of malaria preventive measures (MPM) during pregnancy (Table 1). 
Table 1: The socio-obstetrics of the study women

\begin{tabular}{llll}
\hline Characteristics & Response & Frequency $\mathbf{n = 2 3 0}$ & Percentage \\
\hline Age in years & $<20$ & 4 & 1.7 \\
& $20-35$ & 191 & 83.1 \\
Education status & $>37$ & 35 & 15.2 \\
& Primary & 2 & 0.9 \\
& Secondary & 56 & 24.2 \\
Gravidity & Tertiary & 172 & 74.9 \\
& Primigravida & 84 & 36.4 \\
Place of antenatal care & Multigravida & 92 & 40.3 \\
& Grand multiparous & 54 & 23.4 \\
& Maternity home & 7 & 3.0 \\
& Health Centre & 1 & 0.4 \\
& Private hospital & 11 & 4,8 \\
& General hospital & 3 & 1.3 \\
& Tertiary Health Facility & 201 & 87.0 \\
& Private hospital+Tertiary & 7 & 3.0 \\
& Health Facility & & \\
\hline
\end{tabular}

The most commonly known MPM in pregnancy were the use of anti-malarial prophylaxis (91.8\%) and the use of insecticide treated nets (ITNs) (49.5\%) (Table 2). The majority of the women (70.6\%) used at least one of the malaria preventive measures during pregnancy. About $66.7 \%$ used chemoprophylaxis, while $19.6 \%$ of those that knew of ITNs used it. Among those that used chemoprophylaxis most of them (57.8\%) received single dose of Intermittent Preventive Treatment (IPT) using sulphadoxine-pyrimethamine (SP) during pregnancy, while $37 \%$ received two doses of IPT (Table 3).

Table 2: Knowledge and awareness of malaria preventive measures (MPM) during pregnancy

\begin{tabular}{|c|c|c|c|}
\hline Characteristics & Response & Frequency $(n=230)$ & Percentage \\
\hline \multirow[t]{2}{*}{ Ever heard of MPM } & Yes & 183 & 79.7 \\
\hline & No & 47 & 20.3 \\
\hline \multicolumn{4}{|l|}{ Knowledge of MPM $(n=183)$} \\
\hline Antimalarial prophylaxis & Yes & 168 & 91.8 \\
\hline Use of ITN & Yes & 91 & $49 \cdot 5$ \\
\hline Use of untreated mosquito nets & Yes & 31 & 16.6 \\
\hline Indoor residual spraying & Yes & 5 & 2.7 \\
\hline Environmental sanitation & Yes & 4 & 2.2 \\
\hline
\end{tabular}


Table 3: Use of malaria preventive measures during pregnancy

\begin{tabular}{|c|c|c|c|}
\hline Variable & Response & Frequency $(n=230)$ & Percentage \\
\hline \multirow[t]{2}{*}{ Used MPM } & Yes & 162 & 70.6 \\
\hline & No & 68 & 29,4 \\
\hline \multirow[t]{2}{*}{ Used antimalarial prophylaxis } & Yes & 153 & 66.7 \\
\hline & No & 77 & $33 \cdot 3$ \\
\hline \multirow[t]{3}{*}{ Type of antimalarial prophylaxis $(n=153)$} & 2 doses of IPT & 56 & 37.0 \\
\hline & 1 dose of IPT & 89 & 57.8 \\
\hline & Other antimalarials & 8 & 5.2 \\
\hline \multirow[t]{2}{*}{ Ever heard of ITN } & Yes & 178 & $77 \cdot 5$ \\
\hline & No & 52 & 22.5 \\
\hline \multirow[t]{2}{*}{ Used ITN during pregnancy $(n=178)$} & Yes & 35 & 19.6 \\
\hline & No & 143 & 80.4 \\
\hline
\end{tabular}

A significant association was demonstrated between use of malaria preventive measures and occurrence of neonatal malaria. Mothers that did not use antimalarial prophylaxis or ITN during pregnancy were more likely to give birth to babies with neonatal malaria (Table 4).

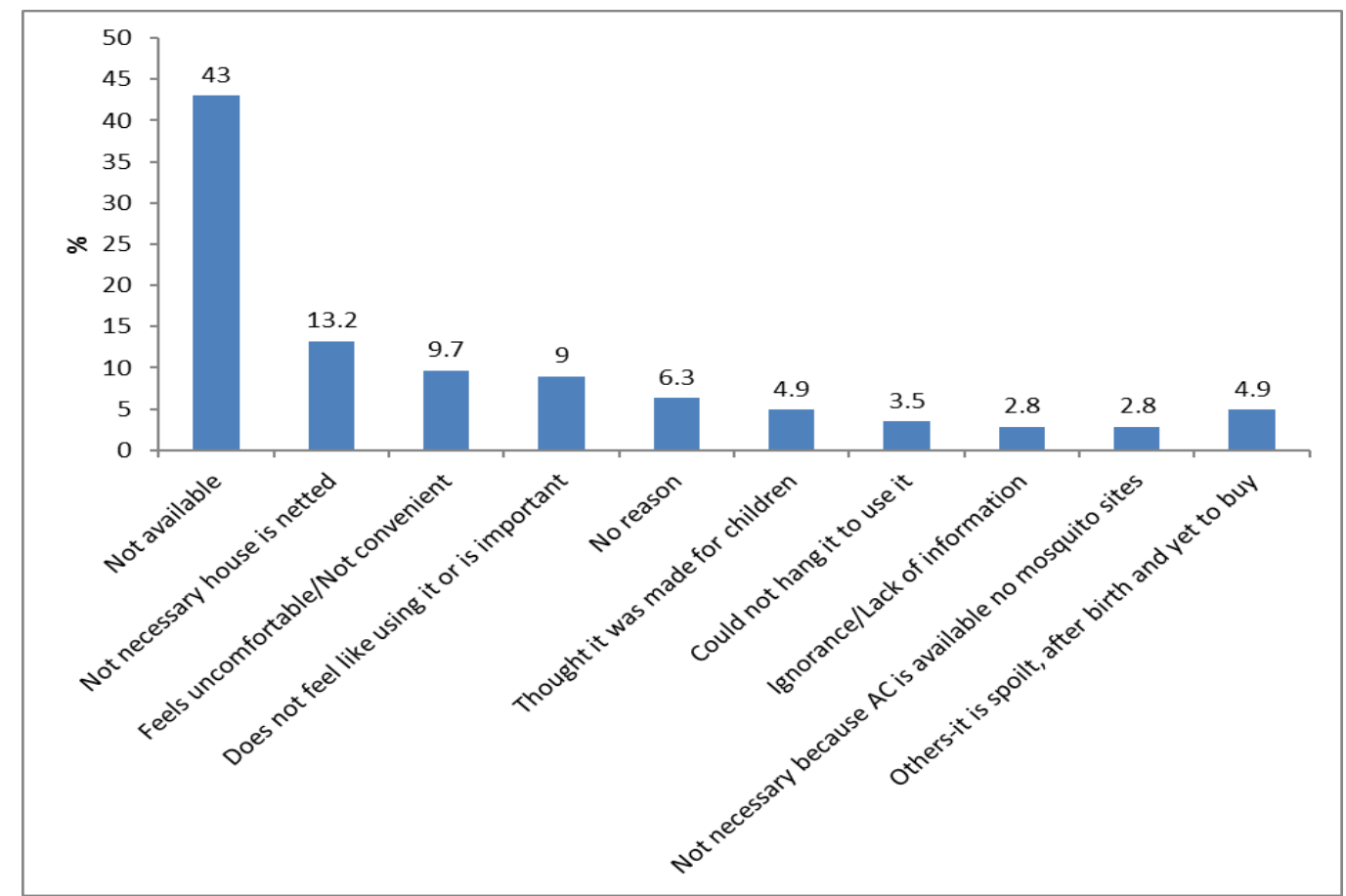

Figure 1: Reasons for not using insecticide treated mosquito nets

Non-availability was the most frequently mentioned reason for not using insecticide treated mosquito nets. Ignorance/lack of information about ITNs and use of air conditioners hence obviating the need for ITNs, were the least reasons given by some participants for not using ITNs (Figure 1). A significant association was demonstrated between non-use of malaria preventive measures and occurrence of neonatal malaria $(p<0.05)$. Majority of mothers that use MPM - 114 (70.6\%), opted for IPT - Intermittent prophylactic treatment of malaria, accounting for 108 of the subjects. Mothers that did not use antimalarial prophylaxis or ITN during pregnancy were more likely to give birth to babies with neonatal malaria (Table 4). 
Table 4: Relationship between the use of malaria preventive measures (MPM) during pregnancy and neonatal malaria

\begin{tabular}{|c|c|c|c|c|c|}
\hline \multirow[t]{2}{*}{ Variable } & \multirow[t]{2}{*}{ Response } & \multicolumn{4}{|c|}{ Neonatal malaria } \\
\hline & & YES; n (\%) & NO; n (\%) & $\mathrm{X}^{2}$ & P-value \\
\hline \multirow[t]{2}{*}{ Use of MPM $(n=230)$} & Yes & $48(29.4)$ & $114(70.6)$ & 8.85 & 0.003 \\
\hline & No & $34(50.0)$ & $34(50.6)$ & & \\
\hline \multirow[t]{2}{*}{ Use of antimalarial prophylaxis $(n=230)$} & Yes & $45(29.2)$ & $108(70.8)$ & 7.85 & 0.005 \\
\hline & No & $37(48.1)$ & $40(51.9)$ & & \\
\hline \multirow{3}{*}{$\begin{array}{l}\text { Pattern of use of antimalarial prophylaxis } \\
(n=153)\end{array}$} & IPT-2 doses & $6(10.5)$ & $50(89.5)$ & & \\
\hline & IPT - 1 dose & $35(39 \cdot 3)$ & $54(60.7)$ & 15.6 & 0.000 \\
\hline & $\begin{array}{l}\text { Other } \\
\text { antimalarials }\end{array}$ & $4(50.0)$ & $4(50.0)$ & & \\
\hline \multirow[t]{2}{*}{ Use of ITN $(n=178)$} & Yes & $4(11.4)$ & $31(88.6)$ & $9 \cdot 52$ & 0.002 \\
\hline & No & $56(38.9)$ & $87(61.1)$ & & \\
\hline
\end{tabular}

\section{Discussion}

Our findings indicate that there was a high knowledge about malaria preventive measures with many women actually using them during pregnancy. This is contrary to the disturbing gap between knowledge and practice previously reported in Western Nigeria (Oladokun et al., 2011). This may not be unconnected with the high level of education of women encountered in this study as most of them had attained tertiary education. Furthermore, the situation may have improved even in areas with contrary reports due to the scaling up of preventive interventions over time.

ITNs have been known to reduce the numbers of infective mosquito bites by 70 to $90 \%$ in various geographical settings (D'Alessandro et al., 1996). In Nigeria, it remains a thing of concern that despite the proven efficacy of this preventive intervention and its supposed availability, malaria still constitutes a serious threat to maternal and neonatal health (Aluko \& Oluwatosin, 2012). Even though, most mothers in this study were aware of the protective nature of ITNs and the fact that mosquito nets have been shown to be effective in the control of malaria in pregnant women, only few reported to use it during pregnancy. This is consistent with previous reports in parts of Kenya where very low usage of ITNs was reported among pregnant (ter Kuile et al., 2003). In contrary, is a recent study in central Tanzania, reported a very high coverage of ITN use among women of reproductive age (Rumisha et al., 2014). The low use of ITN among pregnant women in our study may be associated with other socio-economic factors such as access and perceived cost, discomfort during hot weathers and general nonchalant attitude.

The use of indoor aerosol insecticide sprays was poor in contrast to other reports from south-west Nigeria which showed that the majority of the people used screens and sprays to protect against malaria (Oladokun et al., 2011). The use of aerosol insecticide sprays is very common in Nigeria, the reason however being to curtail the nuisance and controlling lifethreatening mosquitoes. But with the increasing resistance of mosquito populations is threatening the efficacy of those insecticides (Nkya et al., 2013). However, the reported adverse effects and odour of some of the insecticides may be implicated in the decline in their usage. Aerosol insecticide spraying have been recommended but awareness and access to these chemical as well as methods of their application are still low.

Several women in this study used antimalarial prophylaxis. This is in consonance with previous reports where chemoprophylaxis and/or anti-vector measures for prevention of malaria during pregnancy was used with a large proportion combining both methods (Yusuf et al., 2008). It has however been reported that over half of the drugs being used for prophylaxis is not sulfadoxine-pyrimethamine which is currently recommended by the World Health Organization or the Nigerian Federal Ministry of Health (Oladokun et al., 2011). Recommended dosages or 
regimens are not also strictly adhered to. The WHO expected $80 \%$ of all pregnant women living in areas of high transmission to receive IPTp during pregnancy by 2010 (Dare \& Badru, 2001; WHO, 2005). Very low coverage of IPTp has been reported by some studies in Nigeria (Akinleye et al., 2009; Oladokun et al., 2011) and elsewhere in Sub-Saharan Africa (Rumisha et al., 2014). In this study, $57.8 \%$ of the women on prophylaxis used the one dose IPTp to $37 \%$ use of the two doses IPTp. Another consistent study reported only $27.3 \%$ single dose of SP during the index pregnancy (Akinleye et al.,2009). In Kenya, one of the first countries to implement IPTp, the national coverage with two doses of SP was only $4 \%$ five years after IPTp implementation (Greenwood et al., 1994). Malawi has however recorded the highest coverage of $60 \%$ close to achieving the 2000 Abuja target by 2005 (Briand et al., 2007). The models for achieving some of these successes in some endemic countries such as Malawi should be studied and possibly recommended for other countries were success is not being recorded.

The result of this study highlights a high level of awareness on the use of malaria preventive measures by pregnant women though it cannot be adequately associated with deliberate control of neonatal malaria. However, a significant relationship between the use of malaria preventive measures in pregnancy and neonatal malaria was also shown. The increased knowledge is a boost to Global Malaria Control and the roll back malaria strategies aimed at reducing malaria morbidity and mortality among pregnant women. However, the utilization of these measures especially deliberately to control neonatal malaria is contrasting and dependent on varying factors as explained. More efforts need to be made in highlighting the added benefit of using malaria preventive measures during pregnancy as a deliberate strategy to protect the new born against malaria infections. This is very important because this awareness will further motivate pregnant mothers to use preventive measures considering the affection they have for their new-born and their desire to protect them at all cost. There is also need to develop a health promotion package based on factors, especially demystifying the negative perceptions about some preventive measures. This will help increase access to preventive interventions of such.

\section{References}

Akinleye, S.O., Falade, C.O. \& Ajayi, I.O. (2009) Knowledge and utilization of intermittent preventive treatment for malaria among pregnant women attending antenatal clinics in primary health care centers in rural southwest, Nigeria: a cross-sectional study. BMC Pregnancy and Childbirth 9: 28.

Aluko, J.O \& Oluwatosin, A.O. (2012) Utilization of insecticide treated nets during pregnancy among postpartum women in Ibadan, Nigeria: a cross-sectional study. BMC Pregnancy and Childbirth 12: 21.

Briand, V., Cottrell, G., Massougbodji, A. \& Cot, M. (2007) Intermittent preventive treatment for the prevention of malaria during pregnancy in high transmission areas. Malaria Journal 6: 160.

D'Alessandro, U., Ubben, D., Hamed, K., Ceesay, S.J., Okebe, J., Taal, M., Lama, E.K., Keita, M., Koivogui, L., Nahum, A., Bojang, K., Sonko, A.A., Lalya, H.F. \& Brabin, B. (2012) Malaria in infants aged less than six months - is it an area of unmet medical need? Malaria Journal 11:400

D’Alessandro, U., Langerock, P., Bennett, S., Francis, N., Cham, K., \& Greenwood, BM. (1996) The impact of a national impregnated bednet programme on the outcome of pregnancy in primigravidae in Gambia. Transactions of the Royal Society of Tropical Medicine and Hygiene 90, 487-92.

Dare, O.O. \& Badru, O.B. (2001) Promoting the use of insecticide treated materials at the community level: experience from the ITN-Oriade initiative. International Quarterly Community Health Education $\quad 20,281-295$. 
Desai, M., ter Kuile, F.O., Nosten, F., McGready, R., Asamoa, K., Brabin, B. \& Newman, R.D. (2007) Epidemiology and burden of malaria in pregnancy. Lancet Infectious Disease 7 (2): 93-104.

Ekanem, A.D., Anah, M.U. \& Udo, J.J. (2008) The prevalence of congenital malaria among neonates with suspected sepsis in Calabar. Nigeria. Tropical Doctor 38, 73-76.

Gamble, C., Ekwaru, J.P. \& terKuile, F.O. (2006) Insecticide treated nets for preventing malaria in pregnancy. Cochrane Database System Reviews 2: CDo03755.

Greenwood, A., Menendez, C., Alonso, P., Jaffar, S., Langerock, P., Lulat, S., Todd, J., M'Boge, B., Francis, N. \& Greenwood B. (1994) Can malaria chemoprophylaxis be restricted to first pregnancies? Transactions of the Royal Society of Tropical Medicine and Hygiene 88, 681682.

Kevalas, R. (2005) Febrile infant and small child: what solution could be rational? Medicina (Kaunas) 41, 974-87.

Larru, B., Molyneux, E., TerKuile, F.O., Taylor, T., Molyneux, M. \& Terlouw, D.J. (2009) Malaria in infants below six months of age: retrospective surveillance of hospital admission records in Blantyre, Malawi. Malaria Journal 8:310.

Lopez JA, McMillin, K.J., Tobias-Merrill, E.A., Chop, W.M. Jr. (1997) Managing fever in infants and toddlers: toward a standard of care. Postgraduate Medicine 101, 241-2, 245-52.

Menéndez, C., D’Alessandro, U. \& terKuile, F.O. (2007) Reducing the burden of malaria in pregnancy by preventive strategies. Lancet Infectious Disease 7: 126-35.

Nkya, T.E., Akhouayri, I., Kisinza, W., David, J.P. (2013) Impact of environment on mosquito response to pyrethroid insecticides: facts, evidences and prospects. Insect Biochemistry and Molecular Biology 43, 407-416.

Nosten, F., McGready, R. \& Mutabingwa, T. (2007) Case management of malaria in pregnancy. Lancet Infectious Disease 7: 118-25

Oladokun, A., Oladokun, R.E. \& Adesina, O.A. (2011) Knowledge and utilization of malaria control measures by pregnant and newly delivered mothers in Ibadan, Nigeria. African Health Sciences 11, 573-577.

Pusic, M. V. (2007). Clinical management of fever in children younger than three years of age. Paediatrics \& Child Health 12, 469-472.

Rumisha, S.F., Zinga, M.M., Fahey, C.A., Wei, D., Bwana, V.M., Mlozi, M.R.S., Shayo, E.H., Malima, R.C., Mayala, B.K., Stanley, G., Mlacha, T. \& Mboera, L.E.G. (2014) Accessibility, availability and utilisation of malaria interventions among women of reproductive age in Kilosa District in central Tanzania. BMC Health Services Research 14: 452.

Runsewe-Abiodun, I.T., Ogunfowora, O.B., Fetuga, B.M. (2006) Neonatal malaria in Nigeria-a 2year review. BMC Pediatrics 6:19.

Steketee, R.W., Nahlen, B.L., Parise, M.E. \& Menendez, C. (2001) The burden of malaria in pregnancy in malaria-endemic areas. American Journal of Tropical Medicine and Hygiene 64 (Suppl 1-2), 28-35.

ter Kuile, F.O., Terlouw, D.J., Phillips-Howard, P.A., Hawley, W.A., Friedman, J.F., Kariuki, S.K., Shi, Y.P., Kolczak, M.S., Lal, A.A., Vulule, J.M. \& Nahlen, B.L. (2003) Reduction of malaria during pregnancy by permethrin-treated bednets in an area of intense perennial malaria transmission in western Kenya. American Journal of Tropical Medicine and Hygiene 68, 5060

van Eijk, A.M., Hill, J., Larsen, D.A., Webster, J., Steketee, R.W., Eisele, T.P., terKuile, F.O. (2013) Coverage of intermittent preventive treatment and insecticide-treated nets for the control of malaria during pregnancy in sub-Saharan Africa: a synthesis and meta-analysis of national survey data, 2009-11. Lancet Infectious Disease 13, 1029-1042.

Yusuf, O.B., Dada-Adegbola, H.O., Ajayi, I.O. \& Falade, C.O. (2008) Malaria prevention practices among mothers delivering in an urban hospital in southwest Nigeria. Journal of Vector Borne Diseases 45, 217-224. 
WHO (2015) Malaria in high risk groups. World Malaria Report. World Health Organization, Geneva.

WHO (2005) Global Strategy Plan 2005-2015. World Health Organization, Geneva.

WHO (2004) A Strategic Framework for Malaria Prevention and Control During Pregnancy in the African Region. Brazzaville, World Health Organisation. AFR/MAL/04/01 